\title{
Level Crossing Rate and Average Outage Duration of Free Space Optical Links
}

\author{
Chaouki Ben Issaid (http://orcid.org/0000-0002-4481-8168) and Mohamed-Slim Alouini \\ (http://orcid.org/0000-0003-4827-1793) \\ Computer, Electrical, and Mathematical Sciences and Engineering (CEMSE) Division, \\ King Abdullah University of Science and Technology (KAUST), \\ Thuwal, Makkah Province, Saudi Arabia \\ Email: \{chaouki.benissaid, slim.alouini\}@kaust.edu.sa
}

\begin{abstract}
The level crossing rate (LCR) and the average outage duration (AOD) are two important second order statistics that allow a deeper understanding of the behavior of the channel. In this paper, we study these metrics in order to assess the performance of free space optical (FSO) communication links in the presence of weak atmospheric turbulence and Rice-induced pointing errors. More specifically, we derive an integral and a Gauss-Laguerre quadrature representation for both the LCR and the AOD in the single hop case and for their respective bounds in the multihop case. Selected numerical simulations are presented to show the accuracy of the derived results and to study the effect of certain system parameters on these two performance metrics.
\end{abstract}

Index Terms-Level crossing rate, average outage duration, Free-space optical communication, Gauss-Laguerre quadrature, multihop.

\section{INTRODUCTION}

Ultra-reliable low latency communication (uRLLC) services will play a key role in the fifth generation (5G) networks and beyond [1]. In this context, it is important to accurately characterize the average outage duration (AOD) of wireless links. In fact, the temporal variations of the fading channels are characterized by the second order statistics, in particular, the level crossing rate (LCR) and the AOD. These two important statistics are useful for the Markov modeling of wireless channels [2]-[3] and the design of error control codes and the type of diversity to be used in wireless communication systems to combat the effect of fading, and therefore they have been the topic of many works in the literature. Since it can change with time because of user mobility, fading can be represented as a random process. Examining the statistical characteristics of fading time intervals is very critical for designing wireless communications systems. In fact, studying the LCR and the AOD is of major importance when it comes to evaluating adaptive communication systems in terms of the optimum packet length and the packet error rate (PER) [4][5]. Second-order statistics are also more meaningful when analyzing the performance of a communication system in a cellular environment with co-channel interference where the AOD is more important than the probability of outage itself [6]. In [4], the authors derived the expression of the LCR for a multi-antenna system employing maximum ratio

This work was supported by the KAUST Office of Sponsored Research. combining (MRC) in the presence of additive white Gaussian noise and co-channel interferers with unequal received powers and Doppler shifts. The LCR expressions were obtained for both spatially uncorrelated and correlated MRC systems. The authors have investigated, in [7], the PER of interferencelimited mobile packet systems using the Markov model for infinitesimal interval and finite-time interval. The state and state transition probabilities of the Markov chains, used to model the time-varying signal-to-interference ratio (SIR) over frequency-selective fading channels, are obtained from both the LCR and AOD of the SIR. The PER analysis can be carried out for both the cellular communication systems and ad-hoc mobile networks. In [8], the authors derived expressions for the LCR and the AOD for well-known multipath fading models such as Rayleigh, Rice, and Nakagami. They showed that these theoretical expressions present a reasonable fit compared to the measured data. Using Laplace approximation method, Krstić et al. determined the LCR expression of the ratio of product of two $\kappa-\mu$ random variables and Nakagami$\mathrm{m}$ random variables [9]. They also investigated the effect of the fading parameters on the LCR of a wireless relay communication system with two sections operating over $\kappa-\mu$ multipath fading environment in the presence of cochannel interference subjected to Nakagami-m fading. In [10], the authors derived the second order statistics, i.e. the LCR and the AOD, of the received signal envelope and the channel capacity under Inverse Gaussian and Nakagami-Inverse Gaussian fading distributions. They have studied the impact of the distribution parameters on the derived metrics in the context of radio frequency as well as free space optical (FSO) communication systems. Novel exact integral expressions for the LCR and the AOD of $\kappa-\mu$ shadowed fading channels are derived in [11]. The derived expressions provided an accurate representation of the second order statistics of the shadowed fading of empirical data collected for cellular D2D and bodycentric fading channels. Analytical expressions of the LCR and the AOD for the maximal ratio combining (MRC) diversity operating on: (i) non-identical Rayleigh and (ii) independent and identical Ricean were derived in [12]. The authors also presented accurate approximations for both cases in the equal gain combining (EGC) diversity scheme. Yang et al. provided closed-form expressions for the AOD of multihop regenerative communication systems over Rayleigh, 
Nakagami, or Rician fading in [13]. Two type of systems were studied: (i) noise-limited, and (ii) interference-limited systems. In [14], Velkov et al. studied the second order statistics of the amplify-and-forward multihop Rayleigh fading channel. Using the multivariate Laplace approximation theorem, they derived closed-form approximations for the LCR and the AOD. In this work, we derive integral and Gauss Laguerre expressions for the LCR and the AOD of FSO systems in the presence of weak turbulence and under the assumption that the pointing error has non-zero boresight. We also provide bounds for the LCR and AOD in the multihop case.

The reminder of this paper is organized as follows. We start by describing the system model in Section II. We then derive, in Section III, exact integral expressions for the LCR and AOD in our particular set-up. We also give a Gauss-Laguerre quadrature representation for the computation of the LCR. In Section IV, we show some selected numerical simulations to show the accuracy of our approach compared to Monte Carlo (MC) simulations and to investigate the effect of some of the system parameters on both metrics. Finally, the paper ends with a summary of the main results.

\section{SYSTEM MODEL}

In this work, we consider a composite fading model where the fading $h$ can be expressed as $h=h_{l} h_{a} h_{p}$. The path loss $h_{l}$ is assumed to be equal to unity, the random processes $h_{a}$ and $h_{p}$ represent the turbulence fading and the pointing errors, respectively, and are assumed to be independent.

\section{A. Pointing Errors}

Pointing errors are errors due to the misalignment between the transmitter and the receiver. Let $z$ be the distance between the transmitter and the detector, and we assume that the detector is circular with an aperture radius $a$, as shown in Fig. 1.

We consider the complex representation of the displacement as

$$
d(t)=x(t)+j y(t)=r(t) \exp (j \phi(t)),
$$

where $x(t)$ and $y(t)$ are respectively, the horizontal and vertical displacement of the beam in the detector plane. We assume that $x$ and $y$ are independent, and that they have a normal distribution with the same variance $\sigma^{2}$ and different means $\mu_{x}$ and $\mu_{y}$, respectively [15]. Therefore, for a fixed $t$, the radial displacement $r(t)=\sqrt{x(t)^{2}+y(t)^{2}}$ follows a Rice distribution

$$
f_{r}(r)=\frac{r}{\sigma^{2}} \exp \left(-\frac{r^{2}+s^{2}}{2 \sigma^{2}}\right) I_{0}\left(\frac{r s}{\sigma^{2}}\right),
$$

where $s^{2}=\mu_{x}^{2}+\mu_{y}^{2}$ and $I_{0}(\cdot)$ is the $0^{t h}$ order modified Bessel function of the first kind [16, Sec. 8.431.1]. From [17, Sec. 6.6 , we know that $\dot{x}$ is also Gaussian with probability density function (PDF)

$$
f_{\dot{x}}(\dot{x})=\frac{1}{\sqrt{2 \pi \eta^{2}}} \exp \left(-\frac{\dot{x}}{2 \eta^{2}}\right)
$$

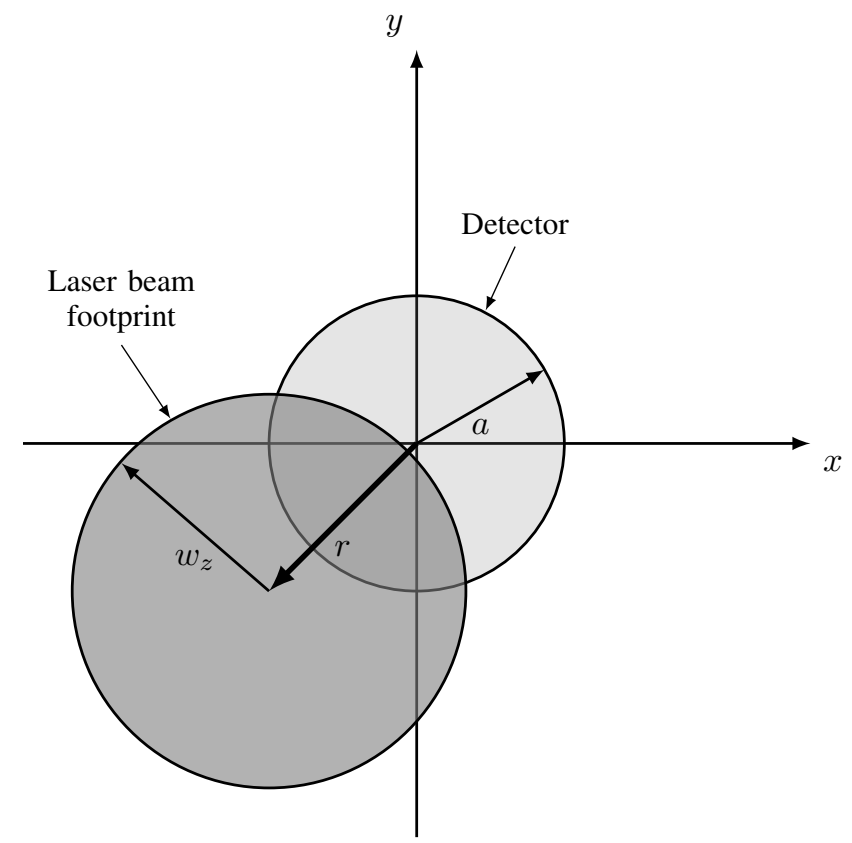

Fig. 1: Illustration of laser beam in the detector plan in the presence of pointing errors

where $\sigma_{\dot{x}}^{2}$ is the variance of the time derivative of $x$. From $[17,6.6-21]$, we have $\mathbb{E}[\dot{x}]=0$ and the variance of $\dot{x}$ is given by $[17,6.6-20]$

$$
\mathbb{V}[\dot{x}]=\eta^{2}=|\ddot{R}(0)|,
$$

where $\ddot{R}(\cdot)$ is the second derivative with respect to (w.r.t) time of the autocorrelation function $R(\cdot)$ of the process $x$. Similarly, we have $\mathbb{E}[\dot{y}]=0$ and $\mathbb{V}[\dot{y}]=\eta^{2}$. In the remainder of this paper, we consider the following generic autocorrelation function [17, Sec 6.5]

$$
R(\tau)=2 \sigma^{2} \exp \left(-\Gamma \frac{|\tau|}{2}\right) \cos (\Omega \tau),
$$

where $\Gamma$ and $\Omega$ are the damping coefficient and the resonance frequency, respectively. In this case, $\eta^{2}=2 \sigma^{2}\left|\Omega^{2}-\frac{\Gamma^{2}}{4}\right|$.

In this work, we assume that the envelope $r$ and the phase $\phi$ are slow variable functions of the time. Following the derivations of [18, Sec. 6.4], the joint distribution of $r$ and $\dot{r}$ is given by [18, Eq. (6-62)]

$$
f_{r \dot{r}}(r, \dot{r})=\frac{r}{\sqrt{2 \pi \eta^{2}} \sigma^{2}} \exp \left(-\frac{r^{2}+s^{2}}{2 \sigma^{2}}\right) \exp \left(\frac{\dot{r}^{2}}{2 \eta^{2}}\right) I_{0}\left(\frac{r s}{\sigma^{2}}\right)
$$

Assuming that the transmitter and the receiver plans are parallel and that the laser beam is perpendicular to the receiver area, the expression of the pointing error loss factor can be approximated by [19] as

$$
h_{p} \simeq A_{0} \exp \left(-\frac{2 r^{2}}{w_{z e q}^{2}}\right),
$$


as long as $\frac{w_{z}}{a}>6$, where $w_{z}$ is the beamwidth, $w_{z e q}$ is the equivalent beam width and $A_{0}$ is the fraction of the collected power at $r=0$. In this case, the distribution of $h_{p}$ is [19]

$$
f_{h_{p}}\left(h_{p}\right)=\frac{\gamma^{2}}{h_{p}} \times\left(\frac{h_{p}}{A_{0}}\right)^{\gamma^{2}}, 0 \leq h_{p} \leq A_{0},
$$

where $\gamma=\frac{w_{z e q}}{2 \sigma}$.

\section{B. Atmospheric Turbulence}

Several models are proposed in the literature to describe the atmospheric turbulence. These models are based on the PDF of the fluctuation of light intensity or irradiance. The lognormal probability density function is the most common ditribution, used in the case of the weak turbulence regime [20]. Therefore, the PDF of $h_{a}$ is given by [19]

$$
f_{h_{a}}\left(h_{a}\right)=\frac{1}{\sqrt{2 \pi} h_{a} \sigma_{R}} \exp \left(-\frac{1}{2}\left(\frac{\log \left(h_{a}\right)-\mu}{\sigma_{R}}\right)^{2}\right),
$$

where $\sigma_{R}^{2}$ is the Rytov variance for a plane wave and the mean is given by $\mu=-\frac{\sigma_{R}^{2}}{2}$.

\section{Multihop Relaying}

The coverage distance of FSO links is quite limited since these links are severely harmed by a path loss. To increase the coverage of these links and enhance their reliability, multihop relaying, where the signal is transmitted by means of intermediate terminals, can reduce the effect of shadowing [21] and enable high data rate transmission [22]. In this section, we consider amplify and forward multihop relaying, which involves the harmonic mean of the instantaneous signal-tonoise ratio of the hops. More specifically, We consider a multihop with $N$ FSO links in cascade for which $h_{a, i}$ is modeled as a lognormal $\mathrm{RV}$, for $i=1, \ldots, N$, with PDF

$$
f_{h_{a, i}}\left(h_{a, i}\right)=\frac{1}{\sqrt{2 \pi} h_{a, i} \sigma_{R, i}} \exp \left(-\frac{1}{2}\left(\frac{\log \left(h_{a, i}\right)-\mu_{i}}{\sigma_{R, i}}\right)^{2}\right),
$$

where $\mu_{i}=-\frac{\sigma_{R, i}^{2}}{2}$ and for $i=1, \ldots, N$, we have

$$
h_{p, i} \simeq A_{0} \exp \left(-\frac{2 r_{i}^{2}}{w_{z e q}^{2}}\right),
$$

where for $i=1, \ldots, N, r_{i}(t)=\sqrt{x_{i}(t)^{2}+y_{i}(t)^{2}}$ follows the Rician PDF

$$
f_{r_{i}}\left(r_{i}\right)=\frac{r_{i}}{\sigma^{2}} \exp \left(-\frac{r_{i}^{2}+s_{i}^{2}}{2 \sigma^{2}}\right) I_{0}\left(\frac{r_{i} s_{i}}{\sigma^{2}}\right),
$$

where $x_{i} \sim \mathcal{N}\left(\mu_{x, i}, \sigma^{2}\right), y_{i} \sim \mathcal{N}\left(\mu_{y, i}, \sigma^{2}\right)$ and $s_{i}^{2}=\mu_{x, i}^{2}+$ $\mu_{y, i}^{2}$, for $i=1, \ldots, N$.

Now, we assume that each fading channel $h_{i}$ can be written as $h_{i}=h_{a, i} h_{p, i}, i=1, \ldots, N$, and let $H$ be defined as

$$
H=\prod_{i=1}^{N} h_{i}=H_{a} H_{p},
$$

where $H_{a}=\prod_{i=1}^{N} h_{a, i}$ and $H_{p}=\prod_{i=1}^{N} h_{p, i}$.

\section{LCR AND AOD COMPUTATION}

The LCR $L_{H}(\cdot)$ and the AOD $T_{H}(\cdot)$ are two important second order statistics that describe the random process behavior. In fact, the LCR represents the average number of crossings of the signal above a certain threshold $h_{0}$ per unit of time and is defined as [18, Eq. (2-73)]

$$
L_{H}\left(h_{0}\right)=\int_{0}^{+\infty} \dot{H} f_{H \dot{H}}\left(h_{0}, \dot{H}\right) d \dot{H} .
$$

The AOD is the average duration during which the signal stays below a certain threshold $h_{0}$ and its expression is given by [18, Eq. (2-75)]

$$
T_{H}\left(h_{0}\right)=\frac{F_{H}\left(h_{0}\right)}{L_{H}\left(h_{0}\right)},
$$

where $F_{H}\left(h_{0}\right)=\mathbb{P}\left(H \leq h_{0}\right)$ is the $\mathrm{CDF}$ of $H$ evaluated at $h_{0}$.

In the remainder of this section, we will be interested in determining the LCR and the AOD of $H$. The CDF of $H$ presents a lower bound for the outage probability of the multihop system (see [23] and Section IV of [24] for more details on how to obtain this lower bound). Since $\left\{h_{a, i}\right\}_{i=1}^{N}$ are lognormal RVs, then $H_{a}$ is also a lognormal RV with PDF

$$
f_{H_{a}}\left(H_{a}\right)=\frac{1}{\sqrt{2 \pi} H_{a} \sigma_{R}} \exp \left(-\frac{1}{2}\left(\frac{\log \left(H_{a}\right)-\mu}{\sigma_{R}}\right)^{2}\right),
$$

where $\sigma_{R}=\sum_{i=1}^{N} \sigma_{R, i}$ and $\mu=\sum_{i=1}^{N} \mu_{i}=-\frac{1}{2} \sum_{i=1}^{N} \sigma_{R, i}^{2}$. As for $H_{p}$, we can re-write its expression as

$$
H_{p}=A_{0}^{N} \exp \left(-\frac{2 \sigma^{2}}{w_{z e q}^{2}} R\right),
$$

where $R=\sum_{i=1}^{N}\left(\frac{r_{i}}{\sigma}\right)^{2}=\sum_{i=1}^{N}\left(\frac{x_{i}}{\sigma}\right)^{2}+\sum_{i=1}^{N}\left(\frac{y_{i}}{\sigma}\right)^{2}$. Since $\left\{\frac{x_{i}}{\sigma}\right\}_{i=1}^{N}$ and $\left\{\frac{y_{i}}{\sigma}\right\}_{i=1}^{N}$ are $2 N$ independent Gaussian RVs with mean $\mu_{x, i}$ or $\mu_{y, i}$ and with unit variance, then the RV $R$ has a noncentral chi-squared distribution

$f_{R}(R)=\frac{1}{2} \exp \left(-\frac{\sigma^{2} R+s^{2}}{2 \sigma^{2}}\right)\left(\frac{\sigma^{2} R}{s^{2}}\right)^{\frac{N-1}{2}} I_{N-1}\left(\frac{s}{\sigma} \sqrt{R}\right)$,

where $s^{2}=\sum_{i=1}^{N} s_{i}^{2}$ and $I_{N-1}(\cdot)$ is the $(N-1)^{t h}$ order modified Bessel function of the first kind [16, Sec. 8.431.1]. Differentiating $R$ w.r.t time, we get

$$
\dot{R}=\frac{2}{\sigma^{2}} \sum_{i=1}^{N} r_{i} \dot{r}_{i} .
$$

Given $\left\{r_{i}\right\}_{i=1}^{N}, \dot{R}$ is a linear combination of $N$ independent Gaussian RVs and thus it is also a Gaussian RV. From [11] and [25], we know that $r_{i}$ and $\dot{r}_{i}$ are independent and $\dot{r}_{i}$ is a zero mean Gaussian RV. Since we are assuming that $x_{i}$ and $y_{i}$ are independent and both have the same variance $\sigma^{2}$, then we have $\sigma_{\dot{x}_{i}}^{2}=\sigma_{\dot{y}_{i}}^{2}$. Thus, the variance of $\dot{r}_{i}$ is given by $\sigma_{\dot{r}_{i}}^{2}=\eta^{2}$, 
for $i=1, \ldots, N$. Given $\left\{r_{i}\right\}_{i=1}^{N}$, the mean and variance of $\dot{R}$ is given by

$$
\begin{aligned}
& \mathbb{E}[\dot{R}]=\frac{2}{\sigma^{2}} \sum_{i=1}^{N} r_{i} \mathbb{E}\left[\dot{r}_{i}\right]=0, \\
& \mathbb{V}[\dot{R}]=\frac{4}{\sigma^{4}} \sum_{i=1}^{N} r_{i}^{2} \mathbb{V}\left[\dot{r}_{i}\right]=\frac{4 \eta^{2}}{\sigma^{2}} R .
\end{aligned}
$$

Therefore, we can write

$$
f(\dot{R} \mid R)=\frac{\sigma}{2 \eta \sqrt{2 \pi R}} \exp \left(-\frac{\sigma^{2} \dot{R}^{2}}{8 \eta^{2} R}\right) .
$$

Since $f_{R \dot{R}}(R, \dot{R})=f(\dot{R} \mid R) f_{R}(R)$, then using (18) and (22) we have

$$
\begin{aligned}
& f_{R \dot{R}}(R, \dot{R})=\frac{\sigma^{N} R^{\frac{N}{2}-1} \exp \left(-\frac{s^{2}}{2 \sigma^{2}}\right)}{4 \eta \sqrt{2 \pi} s^{N-1}} \exp \left(-\frac{\sigma^{2} \dot{R}^{2}}{8 \eta^{2} R}\right) \\
& \times \exp \left(-\frac{R}{2}\right) I_{N-1}\left(\frac{s}{\sigma} \sqrt{R}\right) .
\end{aligned}
$$

Proposition 1. The joint distribution of $H$ and its time derivative $\dot{H}$ is given by (24).

\section{Proof. See Appendix A.}

With the expression of $f_{H \dot{H}}(H, \dot{H})$ at hand, the expression of the LCR is given by Proposition 2.

Proposition 2. The LCR is given by (25).

Proof. See Appendix B.

The expression of $F_{H}\left(h_{0}\right)$ is given by the following corollary

Corollary 1. The CDF of $H$ is given by (26).

Proof. See Appendix C.

Using Gauss-Laguerre quadrature [26, Eq. (22.2.13)], we can approximate the value of LCR in (25) and (15) by

$$
L_{H}\left(h_{0}\right) \approx \frac{w_{z e q}^{N} \exp \left(-\frac{s^{2}}{2 \sigma^{2}}\right)}{2^{\frac{N+5}{2}} \pi \sigma_{R} \sigma^{2} s^{N-1}} \sum_{i=1}^{n} w_{i} g\left(y_{i}\right),
$$

where $y_{i}$ is the $i^{t h}$ root of Laguerre polynomial $L_{n}(y)$ [26, Eq. (25.4.45)], the function $g(\cdot)$ is defined as

$$
\begin{aligned}
& g(y)=y^{\frac{N-1}{2}} e^{\left(1-\gamma^{2}\right) y} \sqrt{8 \eta^{2} y+\beta \sigma_{R}^{2} w_{z e q}^{2} \times} \\
& \exp \left(-\frac{1}{2}\left(\frac{y+\log \left(\frac{h_{0}}{A_{0}^{N}}\right)-\mu}{\sigma_{R}}\right)\right) I_{N-1}\left(\frac{w_{z e q} s}{\sqrt{2} \sigma^{2}} \sqrt{y}\right),
\end{aligned}
$$

and the weight $w_{i}$ is given by [26, Eq. (25.4.45)]

$$
w_{i}=\frac{y_{i}}{(n+1)^{2}\left[L_{n+1}\left(y_{i}\right)\right]^{2}} .
$$

A special case is when $N=1$ which corresponds to a single hop FSO system. The following corollary give the expressions of $f_{h \dot{h}}(\cdot)$, the LCR as well as the CDF of $h$.
Corollary 2. For the single hop case $(N=1)$, the joint distribution of $h$ and its time derivative $h$ is given by (30), the LCR is given by (31), and the CDF of $h$ is given by (32).

\section{Numerical Simulations}

In this section, we investigate the accuracy of the derived results for both the LCR and the AOD by means of MC simulations. A number of samples $M=10^{7}$ has been used to obtain the numerical results. Unless stated otherwise, we consider a dual-hop system, i.e. $N=2$.

We investigate the behavior of the LCR as function of the level $h_{0}$ and for different values of the damping coefficient and resonance frequency in Figures 2 and 3. In fact, we consider a fixed value of the resonance frequency $\Omega=4$ and we plot the LCR for different values of the damping coefficient $\Gamma$ in Fig. 2 . The case $\Gamma=0$ correspond to an oscillatory autocorelation model. The effect of the resonance frequency on the LCR for a fixed damping coefficient $\Gamma=1.5$ is plotted in Fig 3 . An exponential decay autocorrelation model is assumed when $\Omega=0$. We can see that in both plots, the LCR reaches its maximum around the mean value of the process as observed in similar studies done in different context. We studied the effect of the resonance frequency and the damping coefficient on the AOD in Figures 4 and 5. In all these plots, we can see that the derived results match the numerical result obtained using MC simulations. In Fig. 6 , we plotted the LCR for $\Omega=2$ and $\Gamma=1.5$ while varying the number of quadrature points in the Gauss-Laguerre representation. As we can see, as $n$ increases, the representation becomes more accurate and we can see that a relatively small number $n=40$ is sufficient to give a good approximate for the LCR.

In Fig.7 (respectively Fig. 8), we plot the LCR (respectively the AOD) as function of the level $h_{0}$ for different fading environments (i.e. different values of the parameter $s$ ). We can see that for low levels $h_{0}$, the LCR is higher as the value of $s$ is larger, whereas for high levels $h_{0}$, the LCR increases as $s$ becomes smaller. For high levels of $h_{0}$, the AOD of channels with higher values of $s$ is higher than the channels with smaller values of $s$.

The effect of increasing the number of hops on the LCR is studied in Fig. 9. We can see that as the number of hops $N$ increases, the LCR curves are shifted to the right. We also can see that the shape of the LCR is widened for larger values of $N$. Similarly, the AOD of the channels, using the same parameters as in Fig. 9, is depicted in Fig. 10. We note that the AOD curves are monotonically increasing with the level $h_{0}$. Also, the AOD tends to increase with the number of hops. 


$$
\begin{aligned}
& f_{H \dot{H}}(H, \dot{H})=\frac{w_{z e q}^{N+2} \exp \left(-\frac{s^{2}}{2 \sigma^{2}}\right)}{2^{\frac{N+5}{2}} \pi \sigma_{R} \sigma^{2} s^{N-1} H^{2}} \times \\
& \int_{0}^{+\infty} \frac{y^{\frac{N-1}{2}} e^{-\gamma^{2} y} \exp \left(-\frac{w_{z e q}^{2} \dot{H}^{2}}{H^{2}\left[16 \eta^{2} y+2 \beta w_{z e q}^{2} \sigma_{R}^{2}\right]}\right) \exp \left(-\frac{1}{2}\left(\frac{y+\log \left(\frac{H}{A_{0}^{N}}\right)-\mu}{\sigma_{R}}\right)^{2}\right) I_{N-1}\left(\frac{w_{z e q} s}{\sqrt{2} \sigma^{2}} \sqrt{y}\right)}{\sqrt{8 \eta^{2} y+\beta \sigma_{R}^{2} w_{z e q}^{2}}} d y \\
& L_{H}\left(h_{0}\right)=\frac{w_{z e q}^{N} \exp \left(-\frac{s^{2}}{2 \sigma^{2}}\right)}{2^{\frac{N+5}{2}} \pi \sigma_{R} \sigma^{2} s^{N-1}} \times \\
& \int_{0}^{+\infty} y^{\frac{N-1}{2}} e^{-\gamma^{2} y} \sqrt{8 \eta^{2} y+\beta \sigma_{R}^{2} w_{z e q}^{2}} \exp \left(-\frac{1}{2}\left(\frac{y+\log \left(\frac{H}{A_{0}^{N}}\right)-\mu}{\sigma_{R}}\right)^{2}\right) I_{N-1}\left(\frac{w_{z e q} s}{\sqrt{2} \sigma^{2}} \sqrt{y}\right) d y \\
& F_{H}\left(h_{0}\right)=\frac{w_{z e q}^{N+1} \exp \left(-\frac{s^{2}}{2 \sigma^{2}}\right)}{2^{\frac{N}{2}+2} \sqrt{\pi} s^{N-1} \sigma^{2} \sigma_{R}} \int_{0}^{h_{0}} \int_{0}^{+\infty} \frac{e^{-\gamma^{2} y} y^{\frac{N-1}{2}}}{H} \exp \left(-\frac{1}{2}\left(\frac{y+\log \left(\frac{H}{A_{0}^{N}}\right)-\mu}{\sigma_{R}}\right)^{2}\right) I_{N-1}\left(\frac{w_{z e q} s}{\sqrt{2} \sigma^{2}} \sqrt{y}\right) d y d H
\end{aligned}
$$

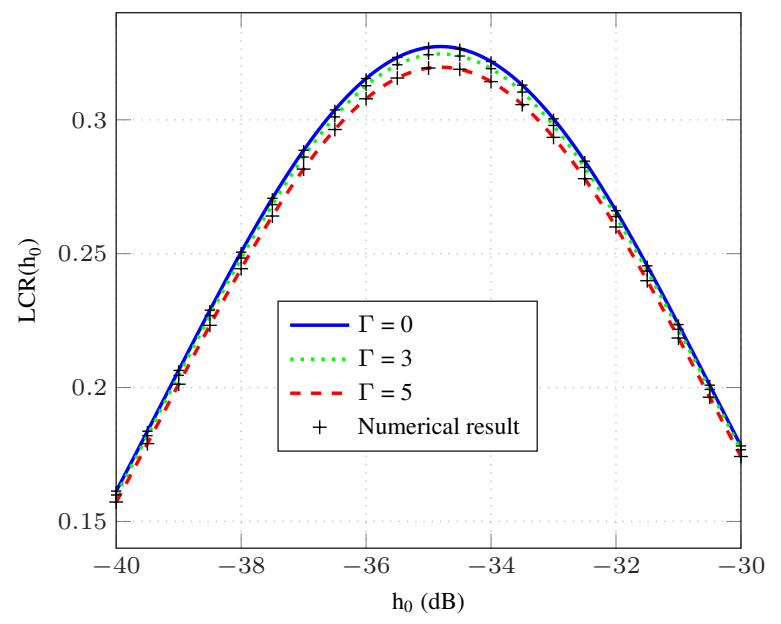

Fig. 2: LCR as function of the level $h_{0}$ for $\Omega=4$ and different values of $\Gamma$.

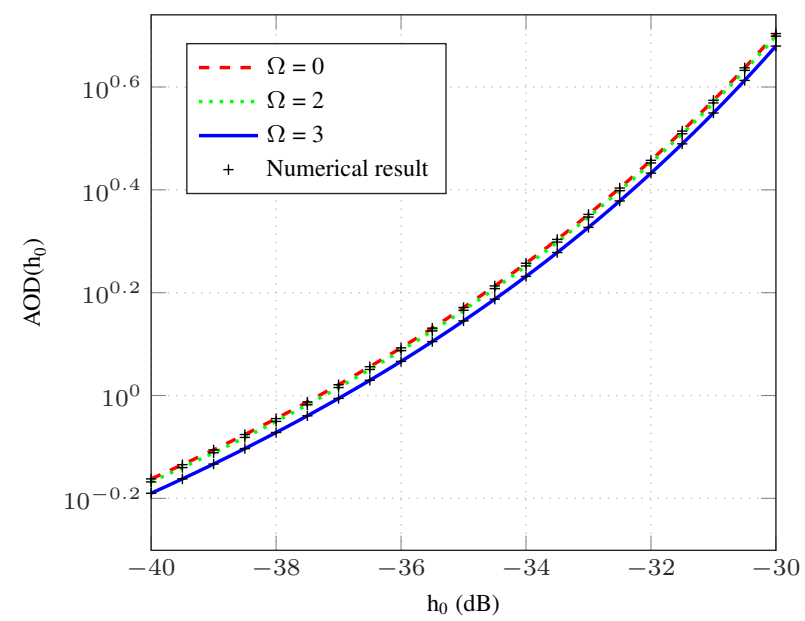

Fig. 5: AOD as function of the level $h_{0}$ for $\Gamma=1.5$ and different values of $\Omega$.

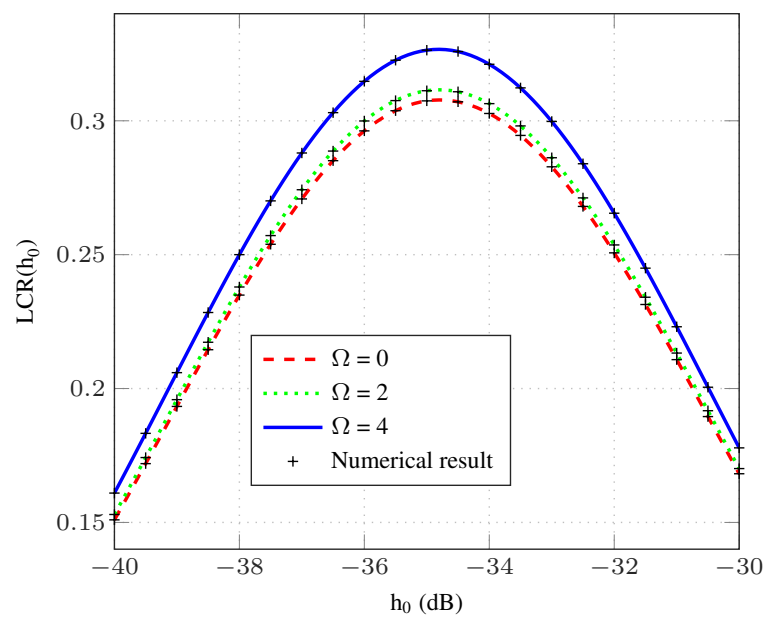

Fig. 3: LCR as function of the level $h_{0}$ for $\Gamma=1.5$ and different values of $\Omega$.

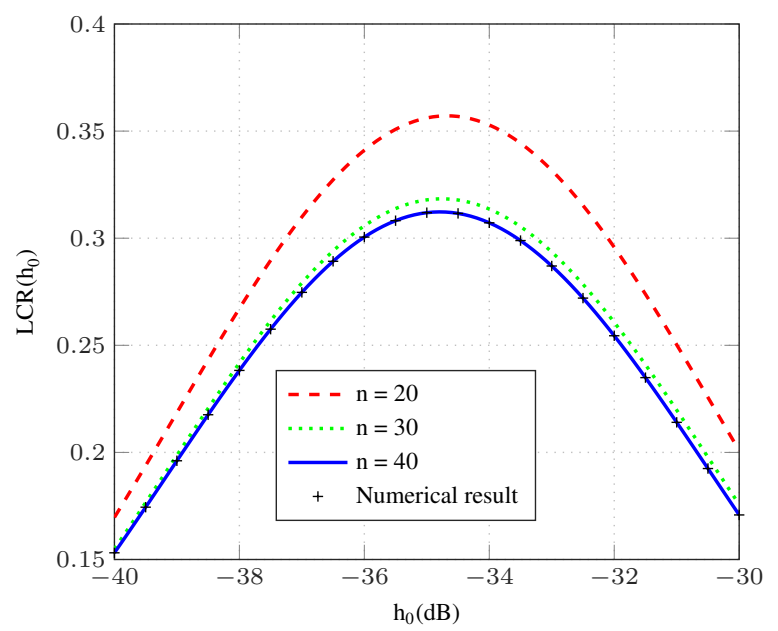

Fig. 6: LCR as function of the level $h_{0}$ for $\Omega=2$ and $\Gamma=1.5$ and different values of $n$. 


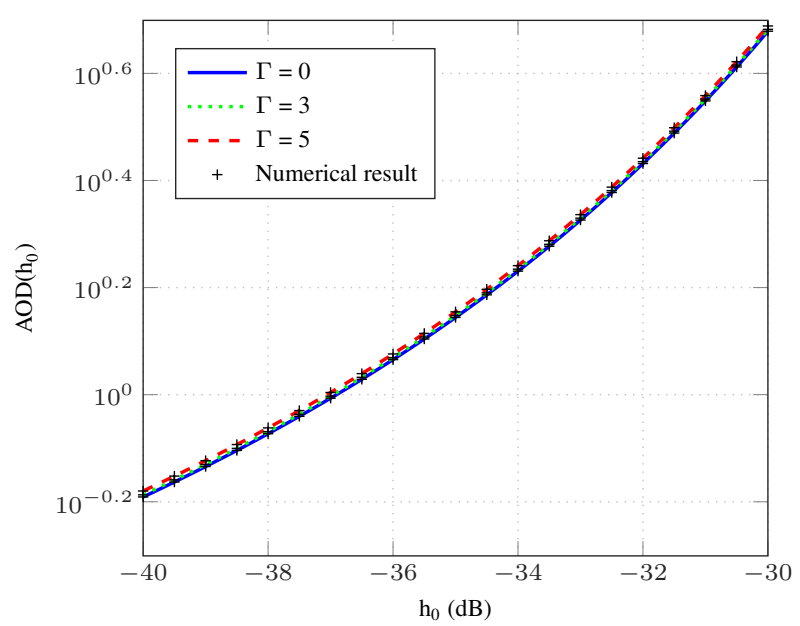

Fig. 4: AOD as function of the level $h_{0}$ for $\Omega=4$ and different values of $\Gamma$.

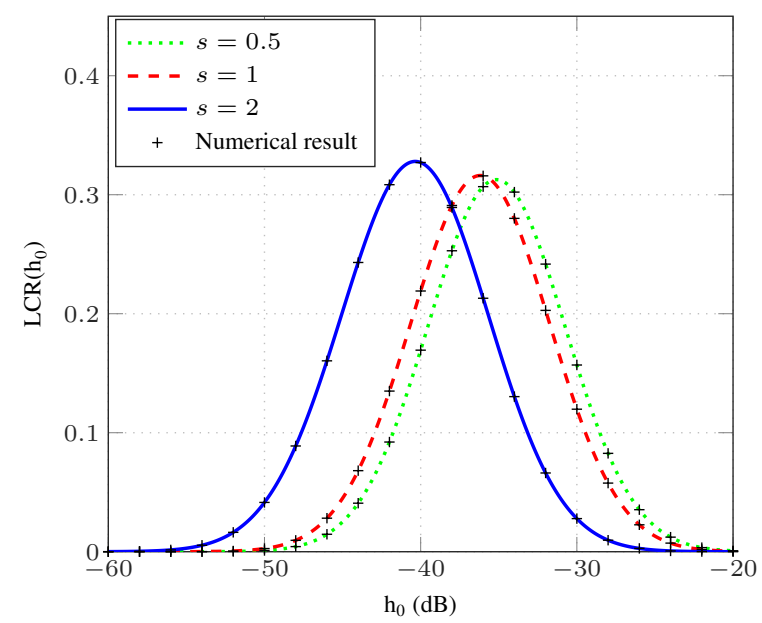

Fig. 7: LCR as function of the level $h_{0}$ for $\Omega=2$ and $\Gamma=1.5$ and different values of $s$.

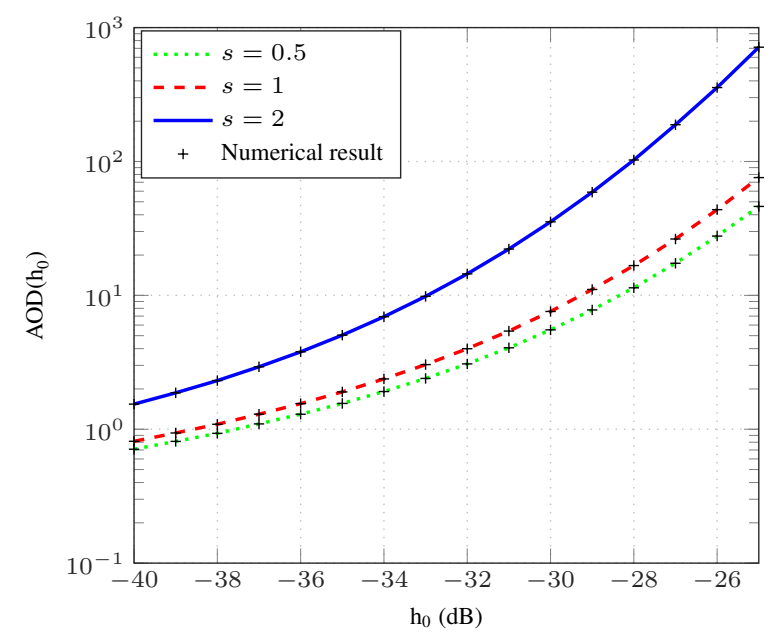

Fig. 8: AOD as function of the level $h_{0}$ for $\Omega=2$ and $\Gamma=1.5$ and different values of $s$.

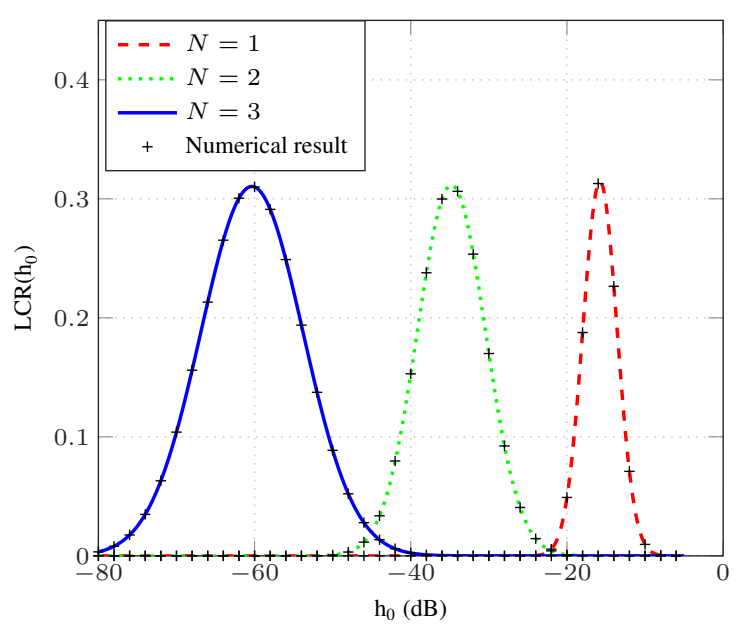

Fig. 9: LCR as function of the level $h_{0}$ for $\Omega=2$ and $\Gamma=1.5$ and different values of $N$.

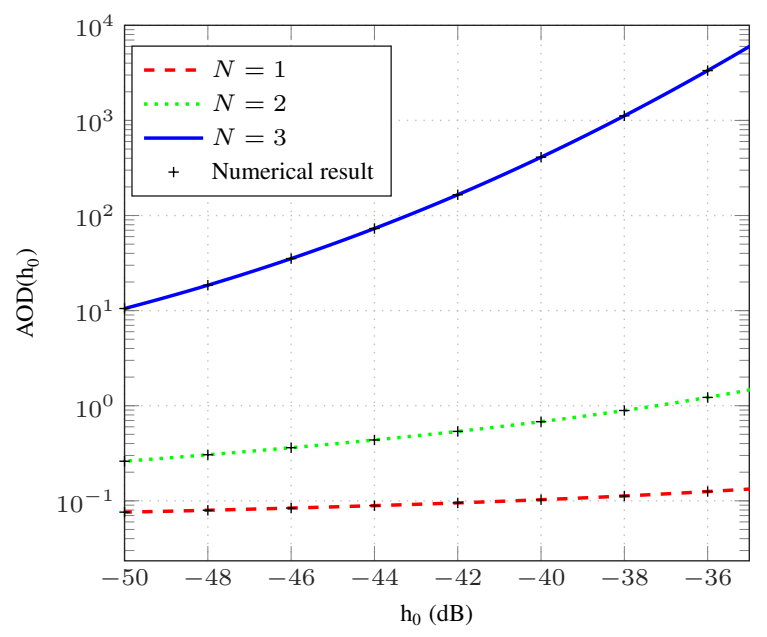

Fig. 10: AOD as function of the level $h_{0}$ for $\Omega=2$ and $\Gamma=1.5$ and different values of $N$.

\section{CONCLUSION}

In this work, we have introduced an integral and a GaussLaguerre representation for the LCR and the AOD of FSO links subject to weak turbulence and in the presence of Rice induced pointing errors. The proposed approach simplified the triple integral expression of the LCR to a finite sum, resulting in a simpler and a faster evaluation of the LCR. We have also provided bounds for the LCR and AOD of the mutihop case. Using these results which were validated with MC simulations, we have investigated the effect of the resonance frequency, the damping coefficient of the radial displacement as well as the number of hops on both the LCR and the AOD.

\section{APPENDIX A}

\section{PROOF OF PROPOSITION 1}

Proof. First, we start by recalling the expression of $R$ as function of $H_{p}$

$$
R=\frac{w_{z e q}^{2}}{2 \sigma^{2}} \log \left(\frac{A_{0}^{N}}{H_{p}}\right)=g\left(H_{p}\right) .
$$




$$
\begin{gathered}
f_{h \dot{h}}(h, \dot{h})=\frac{w_{z e q}^{3} \exp \left(-\frac{s^{2}}{2 \sigma^{2}}\right)}{8 \pi \sigma^{2} \sigma_{R} h^{2}} \times \\
\int_{0}^{+\infty} \frac{e^{-\gamma^{2} y} \exp \left(-\frac{w_{z e q}^{2} \dot{h}^{2}}{h^{2}\left[16 \eta^{2} y+2 \beta w_{z e q}^{2} \sigma_{R}^{2}\right]}\right) \exp \left(-\frac{1}{2}\left(\frac{y+\log \left(\frac{h}{A_{0}}\right)-\mu}{\sigma_{R}}\right)^{2}\right) I_{0}\left(\frac{w_{z e q} s}{\sqrt{2} \sigma^{2}} \sqrt{y}\right)}{\sqrt{8 \eta^{2} y+\beta \sigma_{R}^{2} w_{z e q}^{2}}} d y \\
L_{h}\left(h_{0}\right)=\frac{w_{z e q}}{8 \pi \sigma^{2} \sigma_{R}} \int_{0}^{+\infty} e^{-\gamma^{2} y} \sqrt{8 \eta^{2} y+\beta \sigma_{R}^{2} w_{z e q}^{2}} \exp \left(-\frac{1}{2}\left(\frac{y+\log \left(\frac{h_{0}}{A_{0}}\right)-\mu}{\sigma_{R}}\right)^{2}\right) I_{0}\left(\frac{w_{z e q} s}{\sqrt{2} \sigma^{2}} \sqrt{y}\right) d y \\
F_{H}\left(h_{0}\right)=\frac{w_{z e q}^{2} \exp \left(-\frac{s^{2}}{2 \sigma^{2}}\right)}{2^{\frac{5}{2}} \sqrt{\pi} \sigma^{2} \sigma_{R}} \int_{0}^{h_{0}} \int_{0}^{+\infty} \frac{e^{-\gamma^{2} y}}{H} \exp \left(-\frac{1}{2}\left(\frac{y+\log \left(\frac{H}{A_{0}^{N}}\right)-\mu}{\sigma_{R}}\right) I_{0}\left(\frac{w_{z e q} s}{\sqrt{2} \sigma^{2}} \sqrt{y}\right) d y d H\right.
\end{gathered}
$$

Differentiating w.r.t $t$, we get

$$
\dot{R}=g^{\prime}\left(H_{p}\right) \dot{H}_{p}=-\frac{w_{z e q}^{2} \dot{H}_{p}}{2 \sigma^{2} H_{p}} .
$$

Using the Jacobian, the joint PDF of $H_{p}$ and $\dot{H}_{p}$ can be expressed as function of the joint PDF of $R$ and $\dot{R}$ [27, Chap. 5]

$$
\begin{aligned}
f_{H_{p} \dot{H}_{p}}\left(H_{p}, \dot{H}_{p}\right) & =\left|\begin{array}{cc}
\frac{\partial R}{\partial H_{p}} & \frac{\partial R}{\partial \dot{H}_{p}} \\
\frac{\partial \dot{R}}{\partial H_{p}} & \frac{\partial \dot{R}}{\partial \dot{H}_{p}}
\end{array}\right| f_{R \dot{R}}(R, \dot{R}) \\
& =\left[g^{\prime}\left(H_{p}\right)\right]^{2} f_{R \dot{R}}(R, \dot{R}) .
\end{aligned}
$$

Therefore, we can write

$$
f_{H_{p}, \dot{H}_{p}}\left(H_{p}, \dot{H}_{p}\right)=\left(\frac{w_{z e q}^{2}}{2 \sigma^{2} H_{p}}\right)^{2} f_{R, \dot{R}}(R, \dot{R}) .
$$

With the expression of the joint PDF of $R$ and $\dot{R}$ in (23), we can derive the joint PDF of $H_{p}$ and $\dot{H}_{p}$

$$
\begin{aligned}
& f_{H_{p}, \dot{H}_{p}}\left(H_{p}, \dot{H}_{p}\right)=\frac{w_{z e q}^{N+2} \exp \left(-\frac{s^{2}}{2 \sigma^{2}}\right)}{2^{\frac{N+7}{2}} \sqrt{\pi} \eta \sigma^{2} s^{N-1} H_{p}^{2}}\left[\log \left(\frac{A_{0}^{N}}{H_{p}}\right)\right]^{\frac{N}{2}-1} \\
& \times \exp \left(-\gamma^{2} \log \left(\frac{A_{0}^{N}}{H_{p}}\right)\right) \exp \left(-\frac{w_{z e q}^{2} \dot{H}_{p}^{2}}{16 \eta^{2} H_{p}^{2} \log \left(\frac{A_{0}^{N}}{H_{p}}\right)}\right) \\
& I_{N-1}\left(\frac{s w_{z e q}}{\sqrt{2} \sigma^{2}} \sqrt{\left.\log \left(\frac{A_{0}^{N}}{H_{p}}\right)\right)} .\right.
\end{aligned}
$$

We start by integrating w.r.t $\dot{H}_{a}$

The joint distribution of $H_{a}$ and $\dot{H}_{a}$ is [28]

$$
\begin{aligned}
& f_{H_{a} \dot{H}_{a}}\left(H_{a}, \dot{H}_{a}\right)=\frac{1}{2 \pi \sqrt{\beta}\left(H_{a} \sigma_{R}\right)^{2}} \exp \left(-\frac{1}{2 \beta}\left(\frac{\dot{H}_{a}}{H_{a} \sigma_{R}}\right)^{2}\right) \\
& \times \exp \left(-\frac{1}{2}\left(\frac{\log \left(H_{a}\right)-\mu}{\sigma_{R}}\right)^{2}\right),
\end{aligned}
$$

where $\beta=\left(2 \pi \sigma_{c}\right)^{2}$ and $\sigma_{c}$ is related to the $3-\mathrm{dB}$ cutoff frequency according to $f_{c}=\sqrt{2 \log (2)} \sigma_{c}$.
The joint PDF of $H$ and $\dot{H}$ is derived using the following expression [29]

$$
\begin{aligned}
& f_{H \dot{H}}(H, \dot{H})=\int_{\frac{H}{A_{0}^{N}}}^{+\infty} \int_{-\infty}^{+\infty} \frac{1}{H_{a}^{2}} f_{H_{p} \dot{H}_{p}}\left(\frac{H}{H_{a}}, \frac{\dot{H}}{H_{a}}-\frac{\dot{H}_{a} H}{H_{a}^{2}}\right) \\
& \times f_{H_{a} \dot{H}_{a}}\left(H_{a}, \dot{H}_{a}\right) d \dot{H}_{a} d H_{a} \\
& =\frac{w_{z e q}^{N+2} \exp \left(-\frac{s^{2}}{2 \sigma^{2}}\right)}{2^{\frac{N+9}{2} \pi \sqrt{\beta \pi} \eta \sigma^{2} \sigma_{R}^{2} s^{N-1} H^{2}}} \int_{\frac{H}{A_{0}^{N}}}^{+\infty} \int_{0}^{+\infty} \frac{\left[\log \left(\frac{A_{0}^{N} H_{a}}{H}\right)\right]^{\frac{N}{2}-1}}{H_{a}^{2}} \\
& \times\left(\frac{A_{0}^{N} H_{a}}{H}\right)^{-\gamma^{2}} \exp \left(-\frac{1}{2 \beta}\left(\frac{\dot{H}_{a}}{H_{a} \sigma_{R}}\right)^{2}\right) \times \\
& \exp \left(-\frac{1}{2}\left(\frac{\log \left(H_{a}\right)-\mu}{\sigma_{R}}\right)^{2}\right) \exp \left(-\frac{w_{z e q}^{2}\left(\frac{\dot{H}}{H}-\frac{\dot{H}_{a}}{H_{a}}\right)^{2}}{16 \eta^{2} \log \left(\frac{A_{0}^{N} H_{a}}{H}\right)}\right) \\
& \times I_{N-1}\left(\frac{s w_{z e q}}{\sqrt{2} \sigma^{2}} \sqrt{\log \left(\frac{A_{0}^{N} H_{a}}{H}\right)}\right) d \dot{H}_{a} d H_{a} .
\end{aligned}
$$


where $p=\frac{1}{2 \beta H_{a}^{2} \sigma_{R}^{2}}+\frac{w_{z e q}^{2}}{16 \eta^{2} H_{a}^{2} \log \left(\frac{A_{0}^{N} H_{a}}{H}\right)}$ and $q=$ $\frac{w_{z e q}^{2} \dot{H}}{16 \eta^{2} H H_{a} \log \left(\frac{A_{0}^{N} H_{a}}{H}\right)}$. Using [16, Eq. (3.462.2)], we get

$$
\begin{gathered}
\int_{-\infty}^{+\infty} \exp \left(-\frac{1}{2 \beta}\left(\frac{\dot{H}_{a}}{H_{a} \sigma_{R}}\right)^{2}-\frac{w_{z e q}^{2}\left(\frac{\dot{H}}{H}-\frac{\dot{H}_{a}}{H_{a}}\right)^{2}}{16 \eta^{2} \log \left(\frac{A_{0}^{N} H_{a}}{H}\right)}\right) d \dot{H}_{a} \\
=\frac{\sqrt{\log \left(\frac{A_{0}^{N} H_{a}}{H}\right)} \exp \left(-\frac{w_{z e q}^{2} \dot{H}^{2}}{h^{2}\left[16 \eta^{2} \log \left(\frac{A_{0}^{N} H_{a}}{H}\right)+2 \beta w_{z e q}^{2} \sigma_{R}^{2}\right]}\right)}{\left(4 \sqrt{\beta \pi} H_{a} \sigma_{R} \eta\right)^{-1} \sqrt{w_{z e q}^{2} \beta \sigma_{R}^{2}+8 \eta^{2} \log \left(\frac{A_{0}^{N} H_{a}}{H}\right)}} .
\end{gathered}
$$

Therefore, we get

$$
\begin{aligned}
& f_{H \dot{H}}(H, \dot{H})=\frac{w_{z e q}^{N+2} \exp \left(-\frac{s^{2}}{2 \sigma^{2}}\right)}{2^{\frac{N+5}{2}} \pi \sigma_{R} \sigma^{2} s^{N-1} H^{2}} \times \\
& \int_{\frac{H}{A_{0}^{N}}}^{+\infty} \frac{\left[\log \left(\frac{A_{0}^{N} H_{a}}{H}\right)\right]^{\frac{N-1}{2}} \exp \left(-\frac{1}{2}\left(\frac{\log \left(H_{a}\right)-\mu}{\sigma_{R}}\right)^{2}\right)}{H_{a} \sqrt{w_{z e q}^{2} \beta \sigma_{R}^{2}+8 \eta^{2} \log \left(\frac{A_{0}^{N} H_{a}}{H}\right)}} \\
& \times\left(\frac{A_{0}^{N} H_{a}}{H}\right)^{-\gamma^{2}} I_{N-1}\left(\frac{s w_{z e q}}{\sqrt{2} \sigma^{2}} \sqrt{\log \left(\frac{A_{0}^{N} H_{a}}{H}\right)}\right) d H_{a} .
\end{aligned}
$$

Performing the change of variable $y=\log \left(\frac{A_{0}^{N} H_{a}}{H}\right)$, we get the desired result.

\section{APPENDIX B}

\section{PROOF OF PROPOSITION 2}

Proof. We recall the expression of the average LCR $L_{h}\left(H_{0}\right)$ $L_{H}\left(h_{0}\right)=\int_{0}^{+\infty} \dot{H} f_{H \dot{H}}\left(h_{0}, \dot{H}\right) d \dot{H}=\frac{w_{z e q}^{N+2} \exp \left(-\frac{s^{2}}{2 \sigma^{2}}\right)}{2^{\frac{N+5}{2}} \pi \sigma_{R} \sigma^{2} s^{N-1} h_{0}^{2}} \times$ $\int_{0}^{+\infty} \int_{0}^{+\infty} \frac{\dot{H} y^{\frac{N-1}{2}} e^{-\gamma^{2} y} \exp \left(-\frac{w_{z e q}^{2} \dot{H}^{2}}{H^{2}\left[16 \eta^{2} y+2 \beta w_{z e q}^{2} \sigma_{R}^{2}\right]}\right)}{\sqrt{8 \eta^{2} y+\beta \sigma_{R}^{2} w_{z e q}^{2}}} \times$ $\exp \left(-\frac{1}{2}\left(\frac{y+\log \left(\frac{h_{0}}{A_{0}^{N}}\right)-\mu}{\sigma_{R}}\right) I_{N-1}\left(\frac{w_{z e q} s}{\sqrt{2} \sigma^{2}} \sqrt{y}\right)\right.$

In order to be able to interchange the order of integration, we need to show that the integral w.r.t $\dot{H}$ then w.r.t $y$ is convergent. We start by computing the integral w.r.t $\dot{H}$ to get

$$
\begin{aligned}
& \int_{0}^{+\infty} \dot{H} \exp \left(-\frac{w_{z e q}^{2} \dot{H}^{2}}{h_{0}^{2}\left[16 \eta^{2} y+2 \beta w_{z e q}^{2} \sigma_{R}^{2}\right]}\right) d \dot{H} \\
& =\frac{h_{0}^{2}\left[8 \eta^{2} y+\beta w_{z e q}^{2} \sigma_{R}^{2}\right]}{w_{z e q}^{2}} .
\end{aligned}
$$

Therefore, we need to show that the integral

$$
\begin{aligned}
& I=\int_{0}^{+\infty} y^{\frac{N-1}{2}} e^{-\gamma^{2} y} \sqrt{8 \eta^{2} y+\beta \sigma_{R}^{2} w_{z e q}^{2}} \times \\
& \exp \left(-\frac{1}{2}\left(\frac{y+\log \left(\frac{H}{A_{0}^{N}}\right)-\mu}{\sigma_{R}}\right)^{2}\right) I_{N-1}\left(\frac{w_{z e q} s}{\sqrt{2} \sigma^{2}} \sqrt{y}\right) d y
\end{aligned}
$$

is convergent.

To this end, we introduce the function $\phi(y)=$ $y^{\frac{N-1}{2}} e^{-\gamma^{2} y} \sqrt{8 \eta^{2} y+\beta \sigma_{R}^{2} w_{z e q}^{2}} \quad I_{N-1}\left(\frac{w_{z e q} s}{\sqrt{2} \sigma^{2}} \sqrt{y}\right) \quad$ and we can write

$$
I \leq \int_{0}^{+\infty} \phi(y) d y
$$

Since, we know that:

- $y \mapsto \phi(y)$ is a continuous function on $[0,+\infty[$,

- $\phi(y) \underset{y \rightarrow+\infty}{\sim} \zeta(y)=\frac{2 C_{0} \eta \sqrt{y}}{\sqrt{\pi} \sqrt{\sqrt{y}}} y^{\frac{N}{2}} e^{-\gamma^{2} y} \exp \left(\frac{w_{z e q} s}{\sqrt{2} \sigma^{2}} \sqrt{y}\right)$ (where $C_{0}$ is a constant independent of $y$ and $\left.I_{0}(x) \underset{x \rightarrow+\infty}{\sim} \frac{\exp (x)}{\sqrt{2 \pi x}}[26,(9.7 .1)]\right)$,

- The function $y \mapsto \phi(y)$ has a finite integral over $[0,+\infty[$

$$
\begin{aligned}
& \int_{0}^{+\infty} \phi(y) d y \\
& =\frac{4 \eta C_{0}}{\sqrt{\pi}} \int_{0}^{+\infty} z^{N+\frac{1}{2}} e^{-\gamma^{2} z^{2}} \exp \left(\frac{w_{z e q} s}{\sqrt{2} \sigma^{2}} z\right) d z \\
& =\frac{4 \eta C_{0}}{\sqrt{\pi}}\left(\frac{2 \sigma^{2}}{w_{z e q}^{2}}\right)^{\frac{N}{2}+\frac{3}{4}} e^{\frac{s^{2}}{4 \sigma^{2}}} \Gamma\left(N+\frac{3}{2}\right) D_{-N-\frac{3}{2}}\left(-\frac{s}{\sigma}\right) \\
& <+\infty \text { (using [16, 3.462.1]), }
\end{aligned}
$$

where $D_{\eta}(\cdot)$ is the parabolic cylinder function $[16$, Eq. (9.240)].

Therefore, the integral $I$ is convergent and we can interchange the integration order to obtain

$$
\begin{aligned}
& L_{H}\left(h_{0}\right)=\frac{w_{z e q}^{N} \exp \left(-\frac{s^{2}}{2 \sigma^{2}}\right)}{2^{\frac{N+5}{2}} \pi \sigma_{R} \sigma^{2} s^{N-1}} \int_{0}^{+\infty} y^{\frac{N-1}{2}} e^{-\gamma^{2} y} \times \\
& \sqrt{8 \eta^{2} y+\beta \sigma_{R}^{2} w_{z e q}^{2}} \exp \left(-\frac{1}{2}\left(\frac{y+\log \left(\frac{H}{A_{0}^{N}}\right)-\mu}{\sigma_{R}}\right)\right) \\
& \times I_{N-1}\left(\frac{w_{z e q} s}{\sqrt{2} \sigma^{2}} \sqrt{y}\right) d y .
\end{aligned}
$$

\section{APPENDIX C}

PROOF OF COROLLARY 1

Proof. We start by deriving the PDF of $H_{p}$ from the PDF of $R$. To this end, we recall the expression of $R$

$$
R=\frac{w_{z e q}^{2}}{2 \sigma^{2}} \log \left(\frac{A_{0}^{N}}{H_{p}}\right)=g\left(H_{p}\right)
$$


The PDF of $H_{p}$ is therefore given by

$$
\begin{aligned}
& f_{H_{p}}\left(H_{p}\right)=f_{R}\left(g\left(H_{p}\right)\right)\left|g^{\prime}\left(H_{p}\right)\right| \\
& =\frac{w_{z e q}^{N+1} \exp \left(-\frac{s^{2}}{2 \sigma^{2}}\right)}{2^{\frac{N+3}{2}} s^{N-1} \sigma^{2} H_{p}}\left(\frac{A_{0}^{N}}{H_{p}}\right)^{-\gamma^{2}}\left[\log \left(\frac{A_{0}^{N}}{H_{p}}\right)\right]^{\frac{N-1}{2}} \\
& \times I_{N-1}\left(\frac{s w_{z e q}}{\sqrt{2} \sigma^{2}} \sqrt{\left.\log \left(\frac{A_{0}^{N}}{H_{p}}\right)\right) .}\right.
\end{aligned}
$$

Using [19, Eq. (12)], the PDF of $H$ is

$$
f_{H}(H)=\int_{\frac{H}{A_{0}^{N}}}^{+\infty} \frac{1}{H_{a}} f_{H_{p}}\left(\frac{H}{H_{a}}\right) f_{H_{a}}\left(H_{a}\right) d H_{a} .
$$

Thus, we can write

$$
\begin{aligned}
& f_{H}(H)=\frac{w_{z e q}^{N+1} \exp \left(-\frac{s^{2}}{2 \sigma^{2}}\right)}{2^{\frac{N}{2}+2} s^{N-1} \sqrt{\pi} \sigma_{R} \sigma^{2} H} \int_{\frac{H}{A_{0}^{N}}}^{+\infty} \frac{1}{H_{a}}\left(\frac{A_{0}^{N} H_{a}}{H}\right)^{-\gamma^{2}} \times \\
& {\left[\log \left(\frac{A_{0}^{N} H_{a}}{H}\right)\right]^{\frac{N-1}{2}} \exp \left(-\frac{1}{2}\left(\frac{y+\log \left(\frac{H}{A_{0}^{N}}\right)-\mu}{\sigma_{R}}\right)^{2}\right)} \\
& \times I_{N-1}\left(\frac{s w_{z e q}}{\sqrt{2} \sigma^{2}} \sqrt{\log \left(\frac{A_{0}^{N} H_{a}}{H}\right)}\right) d H_{a} .
\end{aligned}
$$

Performing the change of variable $y=\log \left(\frac{A_{0}^{N} H_{a}}{H}\right)$, the desired result is obtained.

\section{REFERENCES}

[1] M. Bennis, M. Debbah, and H. V. Poor, "Ultrareliable and low-latency wireless communication: Tail, risk and scale," 2018. [Online]. Available: http: //arxiv.org/abs/1801.01270

[2] P. Sadeghi, R. A. Kennedy, P. B. Rapajic, and R. Shams, "Finite-state markov modeling of fading channels - a survey of principles and applications," IEEE Signal Processing Magazine, vol. 25, no. 5, pp. 57-80, September 2008.

[3] W. Wang, H. Yang, and M.-S. Alouini, "Wireless transmission of big data: A transmission time analysis over fading channel," IEEE Transactions on Wireless Cоттиnications, vol. 17, no. 7, pp. 4315-4325, July 2018.

[4] A. O. D. Ali, C. M. Yetis, and M. Torlak, "Novel expressions and applications for the level crossing rate of maximal ratio combining in the presence of cochannel interferers," IEEE Transactions on Vehicular Technology, vol. 66, no. 11, pp. 9793-9808, Nov 2017.

[5] _ - "Optimal packet length for throughput maximization in correlated multi-user channels," in IEEE International Conference on Communications (ICC), May 2016, pp. 1-6.

[6] R. Annavajjala and J. Zhang, "Level crossing rates and average outage durations of SINR with multiple cochannel interferers," in MILITARY COMMUNICATIONS (MILCOM) CONFERENCE, Oct 2010, pp. 1233-1238.

[7] K. Fukawa, H. Suzuki, and Y. Tateishi, "Packet-errorrate analysis using markov models of the signal-tointerference ratio for mobile packet systems," IEEE
Transactions on Vehicular Technology, vol. 61, no. 6, pp. 2517-2530, July 2012.

[8] A. Abdi, K. Wills, H. A. Barger, M.-S. Alouini, and M. Kaveh, "Comparison of the level crossing rate and average fade duration of Rayleigh, Rice and Nakagami fading models with mobile channel data," in 52nd Vehicular Technology Conference (VTC) Fall 2000, vol. 4, 2000, pp. 1850-1857.

[9] D. Krstic, I. Romdhani, M. M. B. Yassein, S. Minic, G. Petkovic, and P. Milacic, "Level crossing rate of ratio of product of two $\kappa-\mu$ random variables and Nakagami-m random variable," in IEEE International Conference on Computer and Information Technology; Ubiquitous Computing and Communications; Dependable, Autonomic and Secure Computing; Pervasive Intelligence and Computing, Oct 2015, pp. 1620-1625.

[10] I. Trigui, A. Laourine, S. Affes, and A. Stephenne, "The inverse Gaussian distribution in wireless channels: Second-order statistics and channel capacity," IEEE Transactions on Communications, vol. 60, no. 11, pp. 3167-3173, November 2012.

[11] S. L. Cotton, "Second-order statistics of $\kappa-\mu$ shadowed fading channels," IEEE Transactions on Vehicular Technology, vol. 65, no. 10, pp. 8715-8720, Oct 2016.

[12] X. Dong and N. C. Beaulieu, Average Level Crossing Rate and Average Fade Duration of Diversity Methods. Boston, MA: Springer US, 2003, pp. 181-203.

[13] L. Yang, M. O. Hasna, and M.-S. Alouini, "Average outage duration of multihop communication systems with regenerative relays," IEEE Transactions on Wireless Communications, vol. 4, no. 4, pp. 1366-1371, July 2005.

[14] Z. Hadzi-Velkov, N. Zlatanov, and G. K. Karagiannidis, "Level crossing rate and average fade duration of the multihop rayleigh fading channel," in IEEE International Conference on Communications, May 2008, pp. 44514455.

[15] F. Yang, J. Cheng, and T. A. Tsiftsis, "Free-space optical communication with nonzero boresight pointing errors," IEEE Transactions on Communications, vol. 62, no. 2, pp. 713-725, February 2014.

[16] I. S. Gradshteyn and I. M. Ryzhik, Table of Integrals, Series, and Products, seventh edition ed. Amsterdam: Elsevier/Academic Press, 2007.

[17] P. Beckmann, Probability in Communication Engineering. Hartcourt Brace \& World,, 1967.

[18] W. C. Lee, Mobile Communications Engineering. McGraw-Hill Professional, 1982.

[19] A. A. Farid and S. Hranilovic, "Outage capacity optimization for free-space optical links with pointing errors," Journal of Lightwave Technology, vol. 25, no. 7, pp. 1702-1710, July 2007.

[20] A. Al-Habash, L. C. Andrews, and R. L. Phillips, "Mathematical model for the irradiance probability density function of a laser beam propagating through turbulent media," Optical Engineering, vol. 40, no. 8, pp. 1554 1562, 2001.

[21] J. Boyer, D. D. Falconer, and H. Yanikomeroglu, "Multi- 
hop diversity in wireless relaying channels," IEEE Transactions on Communications, vol. 52, no. 10, pp. 18201830, Oct 2004.

[22] A. K. Dinnis and J. S. Thompson, "Increasing high data rate coverage in cellular systems using relaying," in IEEE 60th Vehicular Technology Conference, 2004. VTC2004Fall. 2004, vol. 5, Sep. 2004, pp. 3424-3428.

[23] C. B. Issaid, K. Park, M.-S. Alouini, and R. Tempone, "Fast outage probability simulation for FSO links with a generalized pointing error model," in 2016 IEEE Global Communications Conference (GLOBECOM), Dec 2016, pp. 1-7.

[24] C. B. Issaid, K. H. Park, and M.-S. Alouini, "A generic simulation approach for the fast and accurate estimation of the outage probability of single hop and multihop FSO links subject to generalized pointing errors," IEEE Transactions on Wireless Communications, vol. 16, no. 10, pp. 6822-6837, Oct 2017.

[25] W. C. Lee, "Statistical analysis of the level crossings and duration of fades of the signal from an energy density mobile radio antenna," Bell System Technical Journal, vol. 46, no. 2, pp. 417-448.

[26] M. Abramowitz and I. A. Stegun, Handbook of Mathematical Functions with Formulas, Graphs, and Mathematical Tables. New York: Dover, 1972.

[27] H. Kobayashi, B. L. Mark, and W. Turin, Probability, Random Processes, and Statistical Analysis: Applications to Communications, Signal Processing, Queueing Theory and Mathematical Finance. Cambridge University Press, 2011.

[28] T. T. Tjhung and C. C. Chai, "Fade statistics in Nakagami-Lognormal channels," IEEE Transactions on
Communications, vol. 47, no. 12, pp. 1769-1772, Dec 1999.

[29] A. Krantzik and D. Wolf, "Distribution of the fading intervals of modified Suzuki process," in Signal Processing V: Theories and Applications, L. Torres, E. Masgrau, and M. A. Lagunas, Eds. Eds. Amesterdam, The Netherlands: Elsevier Sciences, 1990, pp. 361-364.

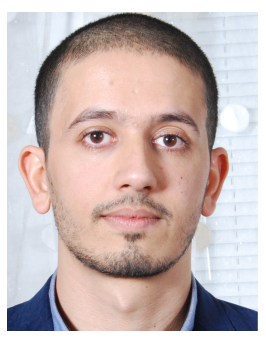

Chaouki Ben Issaid was born in Sfax, Tunisia. He received the Diplôme d'Ingénieur degree from l'École Polytechnique de Tunisie, La Marsa, Tunisia, in 2013. He also holds the Master degree in applied mathematics and computational science from King Abdullah University of Science and Technology (KAUST), Thuwal, Saudi Arabia. Currently, he is working toward the $\mathrm{Ph} . \mathrm{D}$ degree in statistics at KAUST. His current research interests include efficient Monte Carlo simulations for the performance of wireless communication systems.

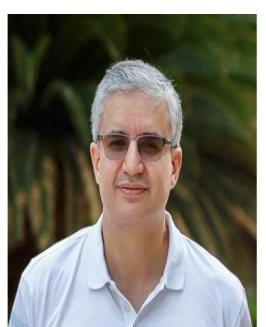

Mohamed-Slim Alouini (S'94-M'98-SM'03-F'09) was born in Tunis, Tunisia. He received the Ph.D. degree in Electrical Engineering from the California Institute of Technology (Caltech), Pasadena, CA, USA, in 1998. He served as a faculty member in the University of Minnesota, Minneapolis, MN, USA, then in the Texas A\&M University at Qatar, Education City, Doha, Qatar before joining King Abdullah University of Science and Technology (KAUST), Thuwal, Makkah Province, Saudi Arabia as a Professor of Electrical Engineering in 2009. His current research interests include the modeling, design, and performance analysis of wireless communication systems. 